\title{
Dendritic Reorganization of Abdominal Motoneurons during Metamorphosis of the Moth, Manduca sexta ${ }^{1}$
}

\author{
R. B. LEVINE ${ }^{2}$ AND J. W. TRUMAN \\ Department of Zoology, University of Washington, Seattle, Washington 98195
}

\begin{abstract}
During metamorphosis in the hawkmoth Manduca sexta, muscles of the abdominal body wall undergo a reorganization. Many die at the end of larval life and are replaced in the adult by newly generated muscles. We have identified several of the motoneurons innervating these muscles and followed them through metamorphosis. The morphology of larval motoneurons is correlated with their target location. Those with medial targets have bilateral dendritic fields, whereas those with lateral targets have dendrites restricted to one side of the segmental ganglion. Some motoneurons innervate the same muscle in all stages of life, but the majority lose their larval targets following entry into the pupal stage. Although some of the latter group also die at this time, most survive to innervate a new adult target. These "respecified" motoneurons undergo a period of dramatic dendritic growth during metamorphosis. The results demonstrate that these identified neurons are capable, under the appropriate conditions of existing in more than one stable morphology.
\end{abstract}

Typically the development of the nervous system does not end with the completion of embryogenesis. A postembryonic phase of development is often required to allow fine tuning of sensory (Hubel et al., 1977) and motor (Brown et al., 1976) systems. Even functioning systems may be modified as animals acquire new behavior as they mature (Nottebohm, 1981). The discrete larval, pupal, and adult stages of the holometabolous insects each have their own characteristic morphology and behavior. Their nervous systems must undergo an extensive reorganization in order to direct the behavior of these radically different stages as the animal progresses through its life history.

In some instances this reorganization during metamorphosis involves the generation of new neurons by retained embryonic neuroblasts (Edwards, 1969). This is particularly true with regard to the major sensory processing areas of the insect brain (Nordlander and Edwards, 1969a, b, 1970; Meinertzhagen, 1973; White and Kankel, 1978; Matsumoto and Hildebrand, 1981), which receive inputs from a large number of imaginal disc-derived sensory neurons. However, the generation of new neurons is not the only strategy employed by

Received October 29, 1984; Revised February 19, 1985; Accepted February 20, 1985

\footnotetext{
${ }^{1}$ This work was supported by grants from the National Institutes of Health and the National Science Foundation to J. W. T., and a postdoctoral fellowship from the National Institutes of Health to R. B. L. We wish to acknowledge the technical assistance of Shirley Reiss, and we thank Barbara Fairbanks for preparing the manuscript.

${ }^{2}$ To whom correspondence should be sent, at his present address: Department of Biology, Rice University, P. O. Box 1892, Houston, TX 77251.
}

the nervous system during metamorphosis. Within the abdominal (Taylor and Truman, 1974; Truman and Reiss, 1976; Levine and Truman, 1982) and thoracic (Casaday and Camhi, 1976) motor centers of the hawkmoth Manduca sexta, motoneurons that were committed to a particular larval function survive metamorphosis to serve as adult motorneurons. The morphology of these neurons as well as their synaptic interactions with other neurons undergo alterations consistent with their new behavioral roles (Levine and Truman, 1982). A similar reuse of neurons appears to occur within the mushroom bodies in the brain of Drosophila (Technau and Heisenberg, 1982)

Many aspects of metamorphic reorganization, such as the growth of new neuronal processes and the formation of new synaptic connections, are reminiscent of events seen during the original embryonic development of the nervous system. During metamorphosis, however, new circuitry must be integrated into an already functioning nervous system, making metamorphosis a particularly relevant model for postembryonic changes in more complex systems. This paper describes the changes that occur within a population of abdominal motoneurons in Manduca. Within this population individual neurons may be identified and their fates during metamorphosis described in detail. Through these studies we hope to relate changes in the structure and synaptic interactions of individual cells to the behavioral changes that the animal shows as it progresses from one stage to another.

\section{Materials and Methods}

Experimental animals. Tobacco hornworms, Manduca sexta (L.), were reared individually on an artificial diet (Bell and Joachim, 1978) under longday conditions (17-hr light/7-hr dark) at $26^{\circ} \mathrm{C}$. The animals pass through five larval instars before entering the pupal stage. All data from larvae were obtained from animals in the second or third day of the fifth instar. Depending upon the rearing conditions, pupae either enter a state of developmental arrest, termed diapause, or initiate adult development which lasts about 18 days under our conditions. Diapausing pupae were used for pupal experiments and adult neurons were stained in insects immediately prior to adult emergence unless otherwise indicated.

Physiology. For intracellular recording from motoneurons the larvae were first anesthetized on ice. An incision was made along the dorsal midline, and the gut was removed. The head and thorax were detached and the abdomen was placed in a Plexiglas recording chamber. The bottom of the chamber contained a square hole which was accessed by air vents. The edges of the square were smeared with stopcock grease (Dow Corning), and the abdomen was pinned open such that the external surfaces of the spiracles were exposed to air while the internal surfaces, including the muscles and nervous system, were exposed to an isotonic saline solution (modified from Weevers, 1966a; KCl, $34 \mathrm{~mm} ; \mathrm{NaCl}, 7 \mathrm{~mm}$; $\mathrm{MgCl}_{2}, 8 \mathrm{~mm}$; $\mathrm{CaCl}_{2}, 14 \mathrm{~mm}$; dextrose, 173 $\mathrm{mM} ; \mathrm{Na} \mathrm{HCO}, 1.25 \mathrm{~mm}$; $\mathrm{Na} \mathrm{H}_{2} \mathrm{PO}_{4}, 1.25 \mathrm{~mm}$ ). The muscles and nervous system were exposed by removing the overiying fat body with care being taken to avoid disruption of the tracheal supply. Good ventilation was essential for continued function of both neurons and muscles.

Abdominal ganglia were elevated on an Insulex-coated metal platform which, being held in a micromanipulator, could be positioned so that the ganglia were stable. Motoneuron somata were impaled with glass microelectrodes which had DC resistances of 25 to 40 megohms when filled with $2 \mathrm{M}$ 
potassium acetate or 80 to 100 megohms when filled with $10 \%$ cobalt nitrate. Electrodes were driven directly through the sheath which surrounds each segmental ganglion. Extracellular records of the motoneurons were obtained by placing suction electrodes on peripheral nerves or upon the surface of muscle fibers. These electrodes were also employed for extracellular stimulation. Recordings from adult nervous systems were obtained with essentially the same techniques, except that the "dorsal pad" of connective tissue which forms during adult development was dissected away from the abdominal ganglia and interganglionic connectives prior to recording.

During the pupal stage the cuticle is rigid and the techniques described above cannot be employed. For recordings from nerve cells in the early pupal stage, a longitudinal strip of cuticle was removed from the dorsal surface of the animal. Then, following removal of the gut and fat body, the remaining body wall was used as a container for saline, and individual ganglia were stabilized as above. With slight modification either dorsal or ventral muscles could be monitored (Bate, 1973a). Near the end of the pupal stage, when adult development was complete, recordings were obtained from "pharate" adults by peeling off the outer covering of dry pupal cuticle and employing the techniques described above for adults.

Motoneurons were identified by correlating intracellular activity with visual inspection of muscle contraction, extra- or intracellular recordings of muscle activity, and extracellular recording of motoneuron axons in the peripheral nerves.

Histology. Neurons were stained by injecting cobalt ions into their somata with microelectrodes using positive current pulses (500 msec duration, 1/ $\mathrm{sec}$, for 15 to $60 \mathrm{~min}$ ). Following reaction with ammonium sulfide (Pitman et al., 1972), the stain was intensified in whole mount (Bacon and Altman, 1977). In addition, motoneuron somata were "backfilled" by dissecting the nervous system free from the animal and draping cut peripheral nerves in a solution of $\mathrm{CoCl}_{2}$ (Truman and Reiss, 1976). Stained cells were photographed or drawn using a drawing tube attachment to a Lietz Ortholux compound microscope. After drawing stained neurons in whole mount, ganglia were reimbeddcd in paraffin and scctioncd at $15 \mu \mathrm{m}$. The sections were then drawn with the aid of a drawing tube attachment.

Motoneuron nomenclature. The naming of motoneurons is complicated by the fact thal some innervale different targets al different stages. Those that innervate the same muscle throughout larval, pupal, and early adult stages were named according to the identity of their target. Those neurons which innervate different targets in larval and adult stages were designated by a number (Taylor and Truman, 1974).

\section{Results}

Background. The fate of the abdominal motoneurons is correlated with the fate of the musculature. In the larva, an abdominal segment such as $\mathrm{A}_{4}$ contains about 50 pairs of muscles that can be divided into internal and external groups (see Figs. 2 and 3). At the start of metamorphosis (i.e., the end of the larval stage), all of the external muscles and some of the internal muscles degenerate, so that only five pairs of internal longitudinal (intersegmental) muscles are present and functional by 2 days into the pupal stage. These internal muscles remain functional through adult development but break down soon after the adult moth emerges from the pupal cuticle (Schwartz and Truman, 1983). In addition, a number of new external muscle groups differentiate during adult development so that at emergence each segment is supplied with about 13 pairs of muscle groups.

Quantitativc changes in the motoneuron population of a segmental abdominal ganglion were first described by backfilling the major peripheral nerves with cobalt at various developmental stages (Taylor and Truman, 1974), and we have used this technique to determine the number of neurons with axons that course in the three subbranches of the "dorsal" segmental nerve (Fig. 1). Whercas the most anterior and posterior of the abdominal ganglia are subject to fusion during metamorphosis, ganglia from segments 3,4 , and 5 remain distinct and the gross morphology of the peripheral nerves remains constant (Taylor and Truman, 1974). Most of the motoneurons revealed by this technique were located in the segment anterior to the filled dorsal nerve branch, although a few were in the same segment.

Waves of cell death follow the larval-to-pupal and pupal-to-adult transitions (Truman, 1983). Thus, the number of motoneurons staining with the backfill procedure declines at these times (Fig. 1). The morphological correlates (Stocker et al., 1978) and the physiological control of this cell death (Truman, 1983; Truman and Schwartz, 1984; Weeks and Truman, 1985) have been described elsewhere. The motoneurons which died after pupal or adult ecdysis innervated muscles that also died at these respective times. As shown below, however, not all motoneurons die following the death of their target.

The majority of the motoneurons having axons which exit the dorsal nerve were individually characterized by intracellular recording and dye injection. Seventeen are excitatory motoneurons that innervate muscles of the body wall. Three others (two in branch $\mathrm{DN}_{\mathrm{a}}$, one in branch DNI) innervate thin muscles associated with the three stretch receptor organs located in each abdominal segment (Weevers, 1966b). Two additional neurons are unpaired and have bifur-
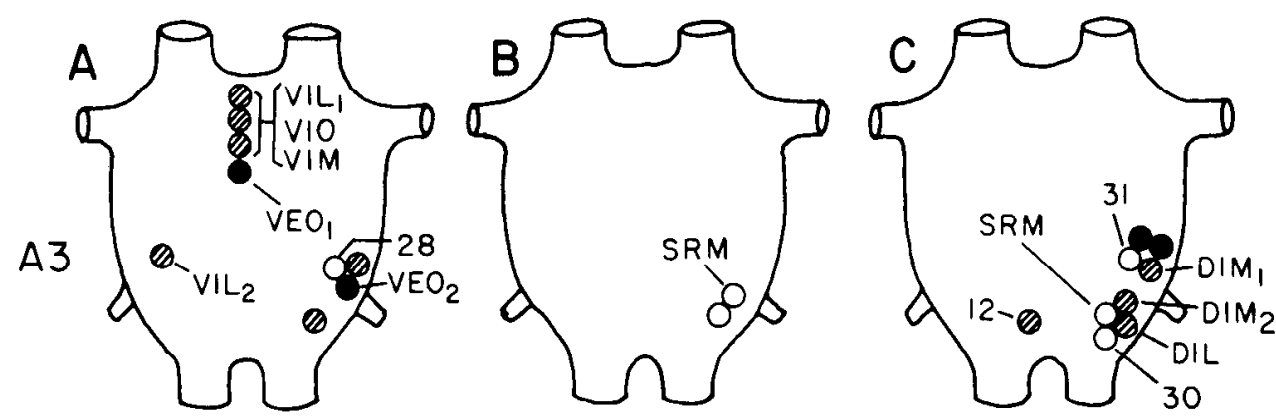

Figure 1. Somata of dorsal nerve motoneurons at different developmental stages. In each case the motoneuron somata which backfill following exposurc of one of the three sub-branches of the dorsal segmental nerve to a cobalt solution are represented. $A$, Motoneurons with axons in the posterior branch of the right dorsal nerve (see arrow) of the fourth abdominal segment. $B$. Anterior branch; $C$, lateral branch. The figure contains information from staining performed in fifth instar lanvae, diapausing pupae, and adults. Solid black circles are used to designate motoneurons which die following pupal ecdysis; stippled circles indicate motoneurons which die after adult emergence; open circles indicate those which are present throughout life. Motoneurons given letter names always innervate the same muscle, whereas those given numbers innervate different targets at different stages (see Figs. 2 and 3 for the locations of target muscles). $S R M$ denotes a motoneuron innervating a muscle associated with one of the stretch receptors. Motoneurons 4 and 5 are ventral unpaired cells with bifurcating axons (see the text). Note that many of the motoneurons have their somata and dendrites located in the segment anterior to their target muscie. 


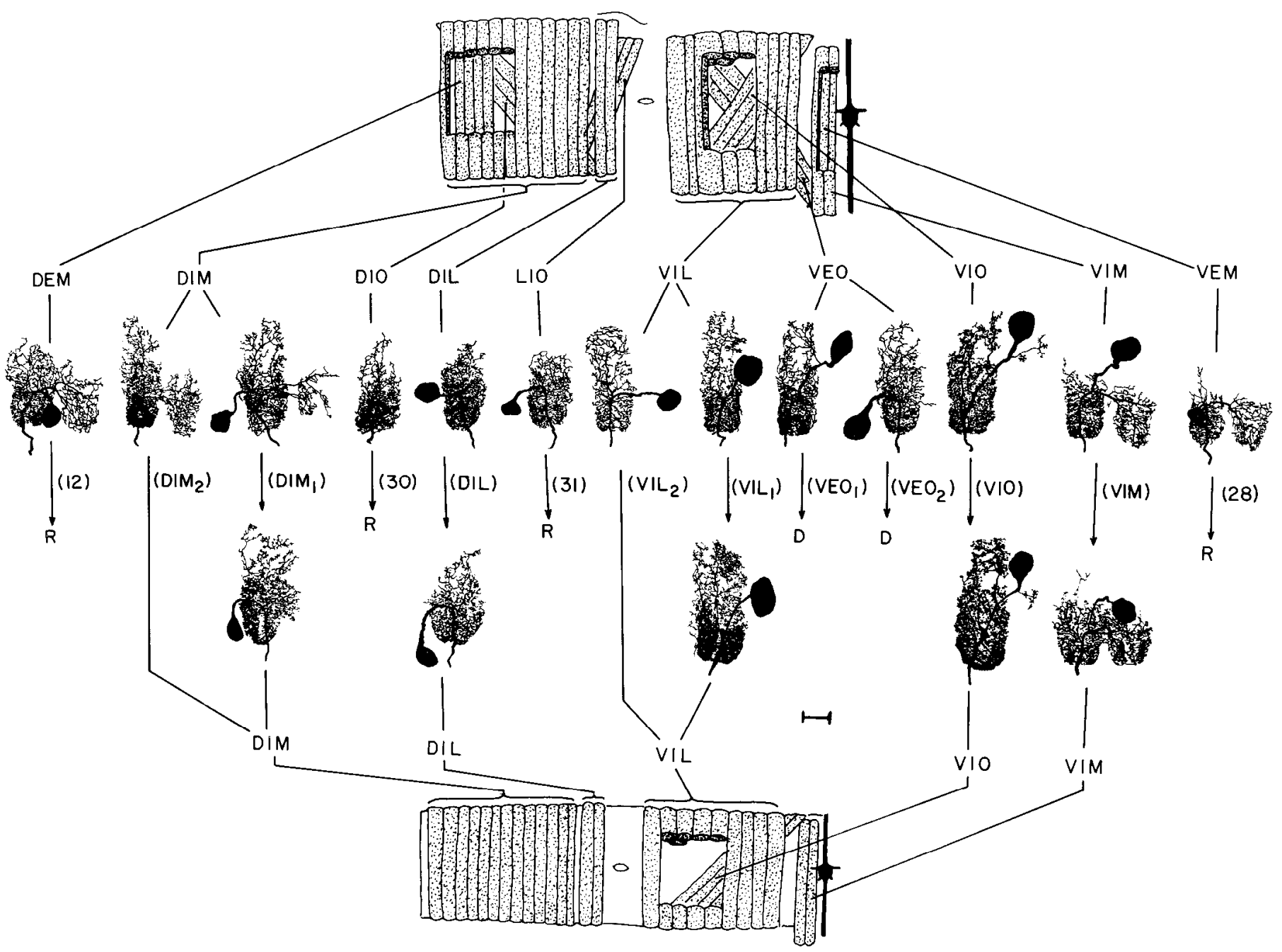

Figure 2. Motoneurons innervating the large intersegmental muscles. The top drawing shows the intersegmental muscles of half of the fourth abdominal segment of a larva. The ventral nerve cord is at the right and the dorsal midline is to the left. The bottom drawing represents the same features in an adult, just prior to emergence (pharate). The upper row of motoneurons innervates the larval muscles as indicated; the lower row represents those present in the pharate adult. Motoneurons were identified and stained by intracellular cobalt injection. The names of the motoneurons indicate target location: $V$, ver irral; $D$, dorsal; $L$, lateral; $M$, medial; I, internal; $E$, external; $O$, oblique. A subscript indicates that two motoneurons innervate the same muscle bundle. Note that the dendritic morphology of the larval motoneurons is correlated with their target location, with motoneurons with medial targets having a bilateral dendritic field. Seven motoneurons innervate the same targets through the pharate stage (five are drawn), after which both neuron and target die. Other motoneurons die following pupal ecdysis $(D)$, and some are respecified $(R)$ to innervate a new target following the death of their larval target. Members of the latter group are shown again in Figure 3. Calibration bar, $50 \mu \mathrm{m}$.

cating axons that enter several sub-branches of the dorsal nerves on each side of the segment. Intracellular recordings from their ventral somata revealed broad, overshooting action potentials unlike the attenuated spikes typically recorded from motoneuron cell bodies (Levine and Truman, 1982). Although they will not be described in detail, both neurons survive metamorphosis and may be analogous to the octopaminergic dorsal unpaired median cells described in locusts (Evans and O'Shea, 1978).

Larval stage motoneurons. The major muscles in the abdomen are the internal muscle groups which span the segment and are primarily longitudinal or oblique in their orientation. These segmentspanning mucles are innervated by motoneurons having somata and dendrites located in the next anterior ganglion (Figs. 2 and $4 A$ ). The axons of these motoneurons descend in the interganglionic connective to the next posterior ganglion and, without entering the neuropil, exit through the dorsal nerve. Several features of the dendritic structure of these neurons are noteworthy. Each has a dense dendritic network in the posterior-dorsal portion of the ganglion, ipsilateral to its axon and target muscle. In addition, some send dendrites into anterior regions of the ganglion (e.g., ventral internal lateral motoneuron (VIL)), and some possess a bilateral dendritic field with branches in the contralateral hemineuropil (e.g., ventral internal medial motoneuron (VIM), Figs. 2 and $4 A$ ). The dendritic structure of these cells is correlated with the location of their target muscle. As seen in Figure 2, motoneurons which innervate muscles along the dorsal or ventral midlines possess bilateral dendritic fields (e.g., VIM and the dorsal external medial motoneuron (DEM)), whereas those that innervate lateral targets have dendrites that are restricted to the hemiganglion ipsilateral to the target (e.g., VIL).

The remaining larval muscles are small external groups which cause relatively minor movements of the body surface. The orientation of these muscles varies from longitudinal to transverse. Those lying in the ventral half of the abdomen are innervated by motoneurons that reside in the ganglion of the same segment and the axons of which course in the ventral nerve (these will be described briefly below). The dorsal external muscles are innervated by motoneurons of the same segmental ganglion with axons in the dorsal nerve (Figs. 2,3 , and $4 B$ ). The external muscle motoneurons differ from the group discussed above not only in terms of target orientation and 


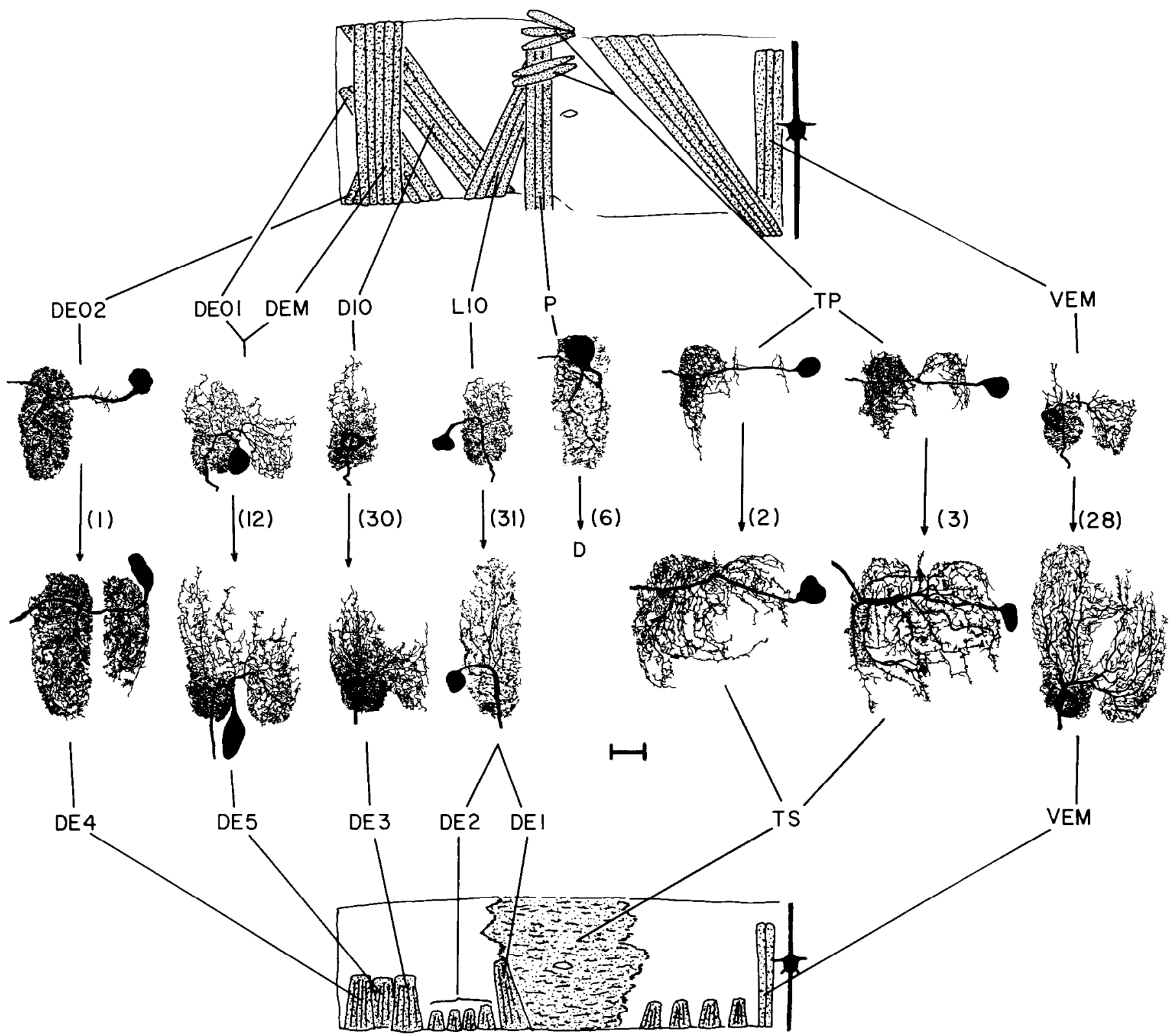

Figure 3. Respecified motoneurons. The top drawing indicates external larval muscles of the fourth abdominal segment that will die following pupal ecdysis. Their adult replacements are showr at the bollom. The motoneurons are shown first in their larval form and, below, in the pharate adult. Note that most of these motoneurons survive the death of their larval target to innervate a newly generated adult muscle. During the pupal stage the motoneurons grow new dendritic processes as the drawings show. Letter names of the muscles refer to their location in the body wall, as described in Figure 2 . $P$, pleural muscles; $T P$, tergopleural; $T S$, tergosternal. Calibration bar, $50 \mu \mathrm{m}$.

axon trajectory, but also with regard to dendritic morphology. Their dendritic processes are typically concentrated in more anterior-dorsal regions of the neuropil (e.g., motoneurons (MN) 2 and 3 ) and do not strictly follow the topographic relationship described above for the internal muscle motoneurons.

Motoneuron fates during metamorphosis. A combination of identification techniques allowed the history of indivudal motoneurons to be traced during metamorphosis. Individual motoneurons were identificd by intraccllular recording and staining in each stage. In most cases the unique combination of soma, peripheral axon, and target positions, combined with a careful comparison of dendritic structures, allowed a relationship between larval, pupal, and adult neurons to be assigned. Furthermore, the somata of some of these motoneurons can bc rccognized unambiguously in sectioned material and were clearly present through each day of adult development (Truman and Reiss, 1976). We have no evidence that new motoneurons are generated in the abdominal ganglia during metamorphosis.
All of the dorsal nerve motoneurons that stain with the backfill procedure in the adult have counterparts in larval ganglia. The larval and adult stages of these cells share identical soma positions, major axon trajectories, and basic dendritic skeleton.

With the exception of very specialized muscles such as the stretch receptor muscles and the spiracular closer muscles, the only muscles to survive after pupal ecdysis were from the internal muscle groups. The motoneurons to these muscles also survived and remained functional throughout adult development and adult emergence, after which both neuron and target died. These neurons showed a slight increase in the extent of dendritic branching during adult development, but their overall morphology remained stable throughout life (Figs. 2 and $4 A$ ). In particular, motoneurons with unilateral dendritic fields did not become bilateral, nor did any of these neurons enter new areas of the neuropil.

All other motoneurons lost their targets either before or slightly after pupal ecdysis. Some then died following the death of their 

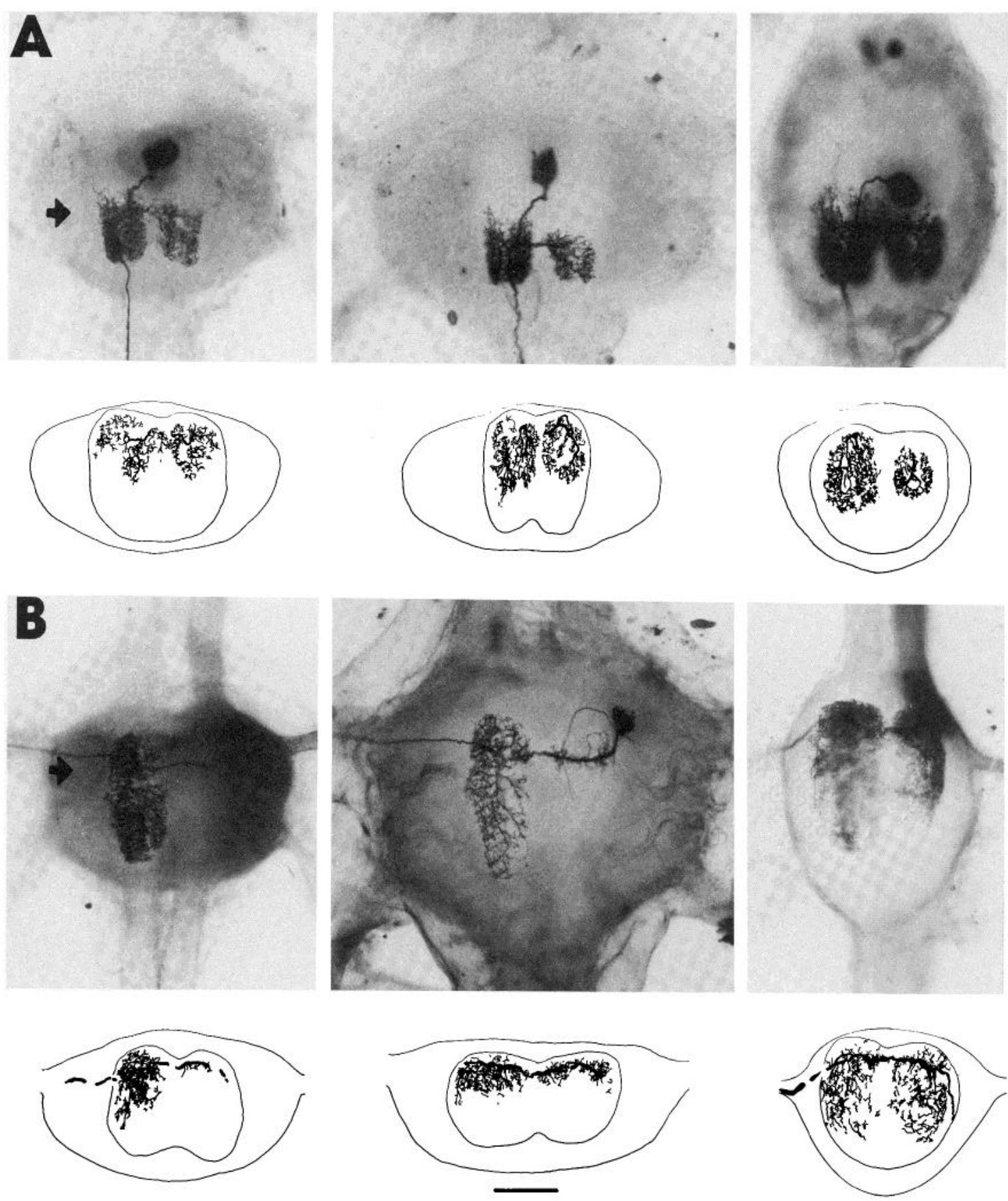

Figure 4. Abdominal motoneurons of the larva (left), diapausing pupa (middle), and pharate adult (right). Motoneurons stained by intracellular cobalt injection were photographed in whole mount, then cross-sectioned. Cross-sections taken at the approximate level indicated by the arrows are shown below each photograph. Calibration bar (bottom), $100 \mu \mathrm{m}$. A, VIM. This motoneuron innervates the ventral internal medial muscle group at each stage. Note its bilateral dendritic field and the axon which descends in the posterior interganglionic connective. Although the basic dendritic shape remains constant, it becomes more dense and projects into more ventral regions during metamorphosis. $B, M N-1$. This motoneuron innervates a dorsal external oblique muscle in the larva and a newly generated dorsal external muscle in the adult. Note that in the diapausing pupa new dendritic branches have started to grow ipsilateral to the soma. In the adult the neuron has a bilateral dendritic field.

target muscles, but the majority survived to innervate new muscles in the adult (Figs. 2 and 3). In each case the adult target was located in the same general area of the body wall as was the larval target, although the orientation was often different. Of the cells that acquired a new peripheral target during metamorphosis, two (MN-2 and MN12) died soon after adult emergence (Truman, 1983). The remainder persisted for the duration of adult life and were responsible for controlling all abdominal movements of the adult. Although these motoneurons were without functional targets early in the pupal stage, they were not quiescent. They retained numerous functional synaptic inputs from the larval stage and their synaptically driven action potentials actively propagated to the periphery (Levine and Truman, 1982).

Each motoneuron which acquired a new target underwent a period of dramatic dendritic growth during adult development (Truman and Reiss, 1976; Levine and Truman, 1982). As described elsewhere (Levine and Truman, 1982), new processes began to appear by about day 2 of the pupal stage (Fig. $4 B$ ), at which point the animals either continued development to the adult or entered an extended period of developmental arrest termed diapause (see "Materials and 

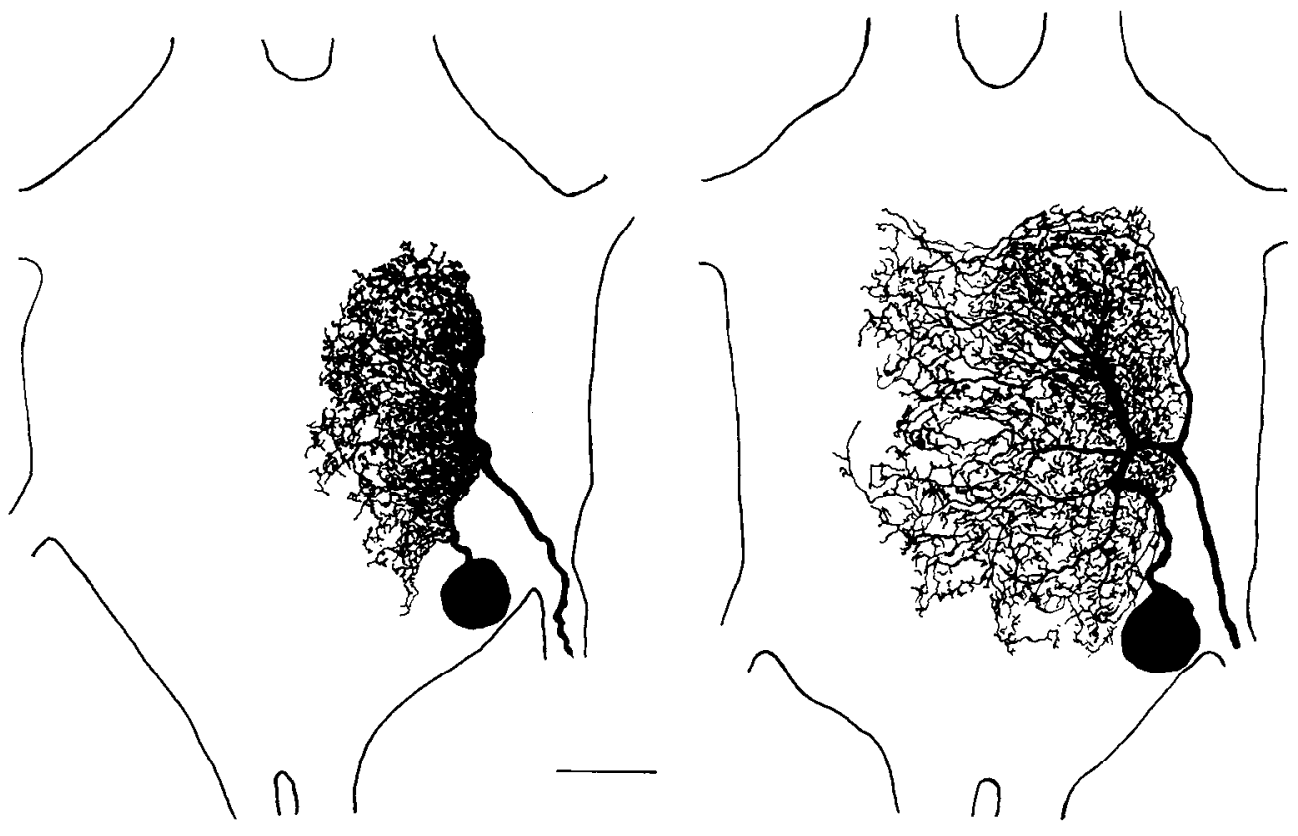

Figure 5. Cobalt backfills of typical larval (left) and adult (right) ventral nerve motoneurons. Although not identified as individuals, backfills of this branch always slain a small population of motoneurons with similar dendritic morphologies. None of the larval neurons have processes crossing the midline of the ganglion, whereas bilateral processes are the rule in the adult. Since all of the larvae muscles innervated by ventral nerve motoneurons die at the end of the larval stage, these neurons must be respecified to innervate a new adult target.
Methods"). In the latter case the cell remained in its pupal form for weeks or months. With the initiation of adult development by the appearance of the steroid hormone 20-hydroxyecdysone (Riddiford and Truman, 1978), the dendritic growth resumed.

The new dendritic growth of these respecified neurons was unique for each cell. In most cases, the adult form of the motoneuron covered a greater expanse of neuropil than did its larval form (Figs. 3 and $4 B$ ). Regardless of the larval morphology of the cell, the dendrites of all surviving adult motoneurons extended throughout the anterior and posterior regions of the neuropil ipsilateral to their axons. In some cases the dendritic growth merely increased the extent of already existing processes (MN-3, MN-28), but in other cases, cells with formerly unilateral dendritic fields extended into contralateral neuropil to form bilateral projections. For example, in the case of $\mathrm{MN}-1$, an existing process contralateral to the axon which was previously devoid of fine branching gave rise to a dense new dendritic network (Figs. 3 and $4 B$; see also Truman and Reiss, 1976; Levine and Truman, 1982). Likewise, MN-30 extended new processes through a posterior commissure into the neuropil contralateral to its axon (Fig. 3).

Ventral nerve motoneurons. The target muscles of all motoneurons with their axons in the ventral nerve die at the end of the larval stage. As was the case with the dorsal nerve motoneurons, many of these ventral nerve cells die following pupal ecdysis (Weeks and Truman, $1984,1985)$, whercas others are retained to innervate newly gener ated muscles. Like their dorsal nerve counterparts, the surviving neurons gain new dendritic processes during adult development (Fig. 5). The acquisition of processes in the hemineuropil contralateral to the axon is particularly striking.

\section{Discussion}

Metamorphosis in the moth, Manduca sexta, brings about a dramatic reorganization of the motor systems that control the behavior of the abdomen. From the larval stage through the end of adult development, major abdominal movements are brought about by the internal muscles and their motoneurons. During this period, the external muscles play a minor role consisting primarily of making postural adjustments of the abdomen and maintaining body tone. At metamorphosis the larval external muscles are completely replaced by sets of longitudinally oriented, adult external muscles. With the subsequent death of the itnernal muscles and their motoneurons after the emergence of the adult, these new external muscles become the sole groups involved in moving the abdomen.
The internal muscles are innervated by the same motoneurons through larval life and up to the time that they die. These motonellrons conserve their dendritic form through metamorphosis, with the shape of the neuron at the time that it dies in the newly emerged adult being essentially identical to its form in the larva. In contrast to the internal muscle motoneurons, those that innervate the external muscles show a very plastic dendritic morphology. Indeed, in many cases the shape of the cell in the adult is markedly different from that seen in the larval stage.

These changes in cell shape in the respecified motoneurons appear to be related to the differences in behavior shown by the adult abdomen as compared to its larval or pupal counterpart. Not only is it involved in certain adult-specific behaviors such as flight and copulation, but unlike the earlier stages, the adult abdomen is relatively incapable of lateral flexion and shows movements confined to the dorsoventral plane. Consequently, the behavioral requirements for the conserved motoneurons are quite different for the larval and adult stages. The dendritic reorganization of the surviving adult motoneurons is consistent with the change in the behavioral role of the abdomen. Neurons that would have functioned antagonistically in the larva during lateral bending movements show synergistic activity in the adult to accomodate the dorsoventral movements. The best studied example of this is cell MN-1 which changes from having a highly lateralized dendritic tree in the larva to showing bilatcrally symmetrical projections in the adult. The bilateral nature of the dendritic fields is correlated with the right and left homologues of the cell sharing numerous common synaptic inputs and firing in synchrony, thereby producing the synchronous contraction of their target muscles during a dorsal flexion (Levine and Truman, 1982). The data reported here for MN-30 and many of the lateral nerve motoneurons indicates that the change from unilateral dendritic arbors to bilateral ones in the adult is a common feature of the reorganization of the abdominal motor system.

It is clear that not all behavioral changes can be related simply to alterations in motoneuron structure. The intersegmental muscle (ISM) motoneurons maintain a relatively constant dendritic structure despite the fact that they do participate in some stage-specific behaviors during the life of the insect (Bate, 1973b; Levine and Truman, 1983). Therefore, important alterations may also occur at the interneuronal level which modify the pattern in which these motoneurons are driven.

Recent work on the central projections of sensory hairs in insects has shown that the projections are somatotopic, with areas on the 
surface of the insect being mapped in a consistent manner in the central neuropil (Murphey, 1981; I evine et al., 1985). In what may be a similar type of arrangement for the motor system, the dendritic morphology of the motoneurons that supply the internal muscles is correlated with the location of their target muscles. Internal muscle motoneurons supplying muscles in the lateral areas of the segment have lateralized dendritic trees ipsilateral to their target, whereas those innervating muscles near the midline have bilateral dendritic projections. Since most of the relevant target muscles span the entire segment, there is no anterior-posterior set of coordinates in this relationship. However, although all internal motoneurons have posterior dendrites, only some send processes into the anterior neuropil. The significance of this fact will remain unclear until we have a better understanding of the neuropil organization. The somatotopic arrangement was evident for the larval internal muscle motoneurons but not for the celis supplying the larval external muscles. This difference probably reflects the fact that the external muscles are much more diverse, with their orientations ranging from longitudinal to transverse. It is of interest that, after metamorphosis, most of the dorsal nerve motoneurons now innervate muscles that are longitudinal in their orientation, and their dendritic trees in the adult are in a somatotopic array.

The respecification of motoneurons during metamorphosis contrasts with the usually permanent commitment of cells to particular fates during development, but it is not unique to insect metamorphosis. In frogs, trigeminal motoneurons in the tadpole are respecified to innervate new adult jaw muscles (Alley and Barnes, 1983; Barnes and Alley, 1983). Comparable transformations of motoneuron morphology and physiology occur during the postembryonic development of the nematode, Caenorhabditis elegans (White et al., 1978). Similarly, identified cells in the locust embryo function first as pioneering guides for axonal growth paths and, later, in a different form, as conventional neurons (Goodman et al., 1981). In each of the invertebrate examples the transformations are confirmed to particular members of the neuronal population and are stereotyped for the individual neuron. In the nervous system of Manduca, as well, only specific motoneurons undergo dendritic growth, and the area of new growth is repeatable for each cell. Structural transformation is therefore a normal component in the life history of certain neurons, and neither the signal for growth nor the response to it is random.

The nature of the signal for dendritic growth is of major interest. One possible influence may be derived from the target muscle. All motoneurons which undergo extensive dendritic growth lose their larval targets, whereas the ISM motoneurons that maintain the same target through metamorphosis have a stable anatomy. The presence of diffcrentiating myoblasts may serve as a signal for the onset of dendritic growth. Alternatively, new synaptic inputs may play an inductive role. Both types of cellular interactions have been implicated in embryonic and postembryonic development of invertebrates (Macagno, 1979; Murphey and Levine, 1980; Schneiderman et al., 1982; Fischbach, 1983; Ho et al., 1983; Nassel and Geiger, 1983; Roederer and Cohen, 1983), but the importance of such interactions for inducing dendritic growth in this system is still unclear.

In their role of directing insect metamorphosis, the insect hormones, ecdysteroid and juvenile hormone, undoubtedly play a major role in directing the shape changes of these motoneurons. However, it is not known whether these hormonal effects occur through a direct action on the neurons or indirectly by altering cues in the extracellular environment. In either case these hormones presumably have a profound effect on neuron form in insects just as steroid hormones influence the dendritic growth of neurons within the vertebrate CNS (Nottebohm, 1980; DeVoogd and Nottebohm, 1981: Gurney, 1981). It is already quite clear that endocrine cues are important for inducing the dendritic reduction and subsequent motoneuron death thal occurs at the end of larval tife (Weeks and
Truman, 1985), as well as the cell death that follows adult emergence (Truman and Schwart7, 1984).

This study provides an indication of the degree to which individual neurons are capable, under the appropriate conditions, of altering their structure and their synaptic interactions with other neurons as their behavioral roles are changing. Although insect metamorphosis may be considered to be a highly specialized process, it is likely that the changes which individual motoneurons display are similar to those which occur in more complex systems during postembryonic maturation.

\section{References}

Alley, K. E., and M. D. Barnes (1983) Birth dates of trigeminal motoneurons and metamorphic reorganization of the jaw myoneural system in frogs. $\mathrm{J}$. Comp. Neurol. 218: 395-405.

Bacon, J. P., and J. S. Altman (1977) A silver intensification method for cobalt filled neurons in whole mount preparations. Brain Res. 138: 359363

Barnes, M. D., and K. I. Alley (1983) Maturation and recycling of trigeminal motoneurons in anuran larvae. J. Comp. Neurol. 218: 406-414.

Bate, C. M. (1973a) The mechanism of the pupal gin trap. I. Segmental gradients and the connections of the triggering sensilla. J. Exp. Biol. 59: 95-107.

Bate, C. M. (1973b) The mechanism of the pupal gin trap. II. The closure movement. J. Exp. Biol. 59: 109-119.

Bell, R. A., and F. A. Joachim (1978) Techniques for rearing laboratory colonies of tobacco hornworms and pink bollworms. Ann. Entymol. Soc. Am. 69: 365-373.

Brown, M. C., J. K. S. Jansen, and D. Van Essen (1976) Polyneuronal innervation of skeletal muscle in new-born rats and its elimination during maturation. J. Physiol. (Lond.) 261: 387-422.

Casaday, G. B., and J. M. Camhi (1976) Metamorphosis of flight motoneurons in the moth Manduca sexta. J. Comp. Physiol. 112: 143-158.

DeVoogd, T., and F. Nottebohm (1981) Gonadal hormones induce dendritic growth in the adult avian brain. Science 214: 202-204.

Edwards, J. S. (1969) Postembryonic development and regeneration of the insect nervous system. Adv. Insect Physiol 6: 97-137.

Evans, P. D., and M. O'Shea (1978) The identification of an octopaminergic neurone and the modulation of a myogenic rhythm in the locust. J. Exp. Biol. 73: 235260.

Fischbach, K. F. (1983) Neural cell types surviving congenital sensory deprivation in the optic lobes of Drosophila melanogaster. Dev. Biol. 95: 1-18.

Goodman, C. S., M. Bate, and N. C. Spitzer (1981) Embryonic development of identified neurons: Origins and transformation of the $\mathrm{H}$ cell. J. Neurosci. 1: $94-102$.

Gurney, M. E. (1981) Hormonal control of cell form and number in the zebra finch song system. J. Neurosci. 1: 658-673.

Ho, R. K., E. E. Ball, and C. S. Goodman (1983) Muscle pioneers: Large mesodermal cells that erect a scaffold for developing muscles and motoneurons in grasshopper embryos. Nature 301: 66-69.

Hubel, D. H., T. N. Wiesel, and S. LeVay (1977) Plasticity of ocular dominance columns in monkey striate cortex. Philos. Trans. R. Soc. Lond. (Biol.) 278: 377-409.

Levine, R. B., and J. W. Truman (1982) Metamorphosis of the insect nervous system: Changes in morphology and synaptic interactions of identified neurons. Nature 299: 250-252.

Levine, R. B., and J. W. Truman (1983) Peptide activation of a simple reflex circuit. Brain Res. 279: 335-338.

Levine, R. B., C. Pak, and D. Linn (1985) The structure, function and metamorphic reorganization of somatatopically projecting sensory neurons in Manduca sexta larvae. J. Comp. Physiol., in press.

Macagno, E. R. (1979) Cellular interactions and pattern formation in the development of the visual system in Daphnia magna. I. Trophic interactions between retinal fibers and laminar neurons. Dev. Biol. 73: 206-238.

Matsumoto, S. G., and J. G. Hildebrand (1981) Olfactory mechanisms in the moth Manduca sexta: Response characteristics and morphology of central neurons in the antennal lobes. Proc. R. Soc. Lond. B. 213: 249-277.

Meinertzhagen, I. A. (1973) Development of the compound eye and optic lobe of insects. In Developmental Neurobiology of Arthropods, D. Young, ed., pp. 51-104, Cambridge University Press, London.

Murphey, R. K. (1981) The structure and development of a somatotopic map in crickets: The cercal afferent projection. Dev. Biol. 88: 236-246.

Murphey, R. K., and R. B. Levine (1980) Mechanisms responsible for changes 
observed in response properties of partially deafferented insect interneurons. J. Neurophysiol. 43: 367-382.

Nassel, D. R., and G. Geiger (1983) Neuronal organization in the fly optic lobes altered by laser ablations early in development or by mutations of the eye. J. Comp. Neurol. 217: 86-102.

Nordlander, R. H., and J. S. Edwards (1969a) Postembryonic brain development in the monarch butterfly Danaus plexippus plexippus L. I. Cellular events during brain morphogenesis. W. Roux Arch. 162: 197-217.

Nordlander, R. H., and J. S. Edwards (1969b) Postembryonic brain development in the monarch butterfly Danaus plexippus plexippus L. II. The optic lobes. W. Roux Arch. 63: 197-220.

Nordlander, R. H., and J. S. Edwards (1970) Postembryonic development in the brain of the monarch butterfly Danaus plexippus plexippus L. III. Development of brain centers other than the optic lobes. W. Roux Arch. 164: $247-260$.

Nottebohm, F. (1980) Testosterone triggers growth of brain vocal control nuclei in adult female canaries. Brain Res. 189: 429-436.

Nottebohm, F. (1981) A brain for all seasons: Cyclical anatomical changes in song control nuclei of the canary brain. Science 214: 1368-1370.

Pitman, R. M. C. D. Tweedle, and M. J. Cohen (1972) Branching of central neurones: Intracellular cobalt injection for light on electron microscopy. Science 176: 412-414.

Riddiford, L. M., and J. W. Truman (1978) Biochemistry of insect hormones and insect growth regulators. in insect Biochemistry, M. Rockstein, ed. pp. 307-357, Academic Press, Inc., New York.

Roederer, E., and M. J. Cohen (1983) Regeneration of an identified central neuron in the cricket. I. Control of sprouting from soma, dendrites, and axon. J. Neurosci. 3: 1835-1847.

Schneiderman, A. M., S. G. Matsumoto, and J. G. Hildebrand (1982) Trans sexually grafted antennae influence the development of sexually dimorphic neurons in the brain of Manduca sexta. Nature 298: 844-846.

Schwartz, L. M., and J. W. Truman (1983) Hormonal control of rates of metamorphic development in the tobacco hornworm Manduca sexta. Dev. Biol. 99: 103-114.
Stocker, R. F., J. S. Edwards, and J. W. Truman (1978) Fine structure of degenerating moth abdominal motorneurons after eclosion. Cell Tissue Res. 191: 317-331.

Taylor, H. M., and J. W. Truman (1974) Metamorphosis of the abdominal ganglia of the tobacco hornworm, Manduca sexta: Changes in populations of identified motorneurons. J. Comp. Physiol. 90: 367-388.

Technau, G., and M. Heisenberg (1982) Neural reorganization during metamorphosis of the corpora pedenculata in Drosophila melanogaster. Nature 295: 405-407.

Truman, J. W. (1983) Programmed cell death in the nervous system of an adult insect. J. Comp. Neurol. 216: 445-452.

Truman, J. W., and S. E. Reiss (1976) Dendritic reorganization of an identified motorneuron during metamorphosis of the tobacco hornworm moth. Science 192: 477-479

Truman, J. W., and L. M. Schwartz (1984) Steroid regulation of neuronal death in the moth nervous systom. J. Neurosci. 4: 274280.

Weeks, J. C., and J. W. Truman (1984) Neural organization of peptideactivated behaviors during the metamorphosis of Manduca sexta. II. Retention of the proleg motor pallern despile the loss of the prolegs at pupation. J. Comp. Physiol. 155: 423-433.

Weeks, J. C., and J. W. Truman (1985) Independeñt steroid control of the tates of motoneurons and their muscles during insect metamorphosis. $\mathrm{J}$. Neurosci, in press.

Weevers, R. deG. (1966a) A lepidopteran saline: Effects of inorganic cation concentrations on sensory, reflex and motor responses in a herbivorous insect. J. Exp. Biol. 44: 163-175.

Weevers, R. deG. (1966b) The physiology of a lepidopteran muscle receptor I. The sensory response to stretching. J. Exp. Biol. 44: 177-194.

White, J. G., D. G. Albertson, and M. A. R. Anness (1978) Connectivity changes in a class of motoneuron during the development of a ncmatode. Nature 271: 764-766.

White, K., and D. R. Kankel (1978) Patterns of cell divisions and movement in the formation of the imaginal nervous system in Drosophila melanogaster. Dev. Biol. 65: 296-321. 